\title{
Trends in the exchange current for hydrogen evolution
}

Nørskov, Jens Kehlet; Bligaard, Thomas; Logadottir, Ashildur; Kitchin, J.R.; Chen, J.G.; Pandelov, S.; Stimming, $U$.

Published in:

Journal of The Electrochemical Society

Link to article, DOI:

$10.1149 / 1.1856988$

Publication date:

2005

Document Version

Publisher's PDF, also known as Version of record

Link back to DTU Orbit

Citation (APA):

Nørskov, J. K., Bligaard, T., Logadottir, A., Kitchin, J. R., Chen, J. G., Pandelov, S., \& Stimming, U. (2005).

Trends in the exchange current for hydrogen evolution. Journal of The Electrochemical Society, 152(2), J23-J26. https://doi.org/10.1149/1.1856988

\section{General rights}

Copyright and moral rights for the publications made accessible in the public portal are retained by the authors and/or other copyright owners and it is a condition of accessing publications that users recognise and abide by the legal requirements associated with these rights.

- Users may download and print one copy of any publication from the public portal for the purpose of private study or research.

- You may not further distribute the material or use it for any profit-making activity or commercial gain

- You may freely distribute the URL identifying the publication in the public portal 


\title{
Trends in the Exchange Current for Hydrogen Evolution
}

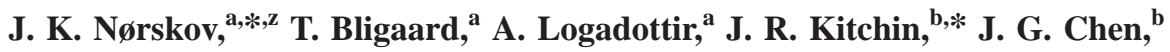 \\ S. Pandelov, ${ }^{\mathrm{c}}$ and U. Stimming ${ }^{\mathrm{c}, *}$ \\ ${ }^{a}$ Department of Physics, Center for Atomic-scale Materials Physics, Technical University of Denmark, \\ DK-2800 Lyngby, Denmark \\ ${ }^{b}$ Department of Chemical Engineering, University of Delaware, Newark, Delaware 19716, USA \\ ${ }^{c}$ Department of Physics, Technical University Munich, D-85748 Garching, Germany
}

\begin{abstract}
A density functional theory database of hydrogen chemisorption energies on close packed surfaces of a number of transition and noble metals is presented. The bond energies are used to understand the trends in the exchange current for hydrogen evolution. A volcano curve is obtained when measured exchange currents are plotted as a function of the calculated hydrogen adsorption energies and a simple kinetic model is developed to understand the origin of the volcano. The volcano curve is also consistent with Pt being the most efficient electrocatalyst for hydrogen evolution.

(C) 2005 The Electrochemical Society. [DOI: 10.1149/1.1856988] All rights reserved.
\end{abstract}

Manuscript submitted May 10, 2004; revised manuscript received August 12, 2004. Available electronically January $24,2005$.

The hydrogen evolution reaction is one of the most important electrochemical reactions, whereby protons from solution combine with electrons at an electrode to form first hydrogen atoms chemisorbed at the electrode surfaces and then $\mathrm{H}_{2}$ gas. The ability of a given metal to catalyze the hydrogen evolution reaction is usually measured by the exchange current density, which is the rate of hydrogen evolution per surface area at the electrode potential, where the reaction is at equilibrium.

Different materials have widely different exchange current densities. In this paper, we investigate the origin of these variations. We calculate the chemisorption energy for hydrogen for a number of metals. We also collect a number of measured exchange current densities for the same metals and show a volcano curve when the measured currents are plotted as a function of the calculated hydrogen adsorption energies. We show that we are able to understand the variation in the exchange current semi-quantitatively using a simple kinetic model. We also show that the fact that the Pt group metals are best for hydrogen evolution follows directly from the thermochemistry of the reaction. Finally, we discuss the relationship of the volcano curve presented here to other volcano curves introduced previously. ${ }^{1,2}$

\section{Database for Hydrogen Chemisorption Energies}

The starting point for our discussion is a consistent set of hydrogen chemisorption energies. We have obtained these from density functional theory (DFT) calculations. The electronic structure problem has been solved using a plane wave pseudopotential implementation, ${ }^{3,4}$ employing the ultrasoft pseudopotentials of Vanderbilt ${ }^{5}$ to represent the ionic cores. All calculations were performed with the RPBE exchange-correlation functional ${ }^{6}$ on periodically repeated metal slabs.

The surface coverage of the adsorbates was $1 / 4$ or 1 monolayer. In both cases the integral adsorption energy is defined by

$$
\Delta E_{\mathrm{H}}=\frac{1}{\mathrm{n}}\left(E(\operatorname{surf}+n H)-E(\operatorname{surf})-\frac{\mathrm{n}}{2} E\left(H_{2}\right)\right)
$$

where $n$ is the number of $\mathrm{H}$ atoms in the calculation. We have used a $2 \times 2$ surface cell for all calculations, and for this unit cell, $n=1$ corresponds to a coverage of $1 / 4$, while $n=4$ corresponds to a coverage of 1 . Unless otherwise noted, all calculations were done on a three layer fcc(111) slab at the RPBE lattice constant separated by five equivalent layers of vacuum. Adding a fourth layer changes the hydrogen binding energy on $\operatorname{Pt}(111)$ by $0.01 \mathrm{eV}$. The bottom two layers were fixed, and the top layer was allowed to

\footnotetext{
* Electrochemical Society Active Member.

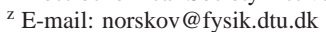

relax. The adsorbate was in the fcc site. A $4 \times 4 \times 1$ MonkhorstPack k-point setup was used, with maximum symmetry applied to reduce the number of k-points in the calculations. The dipole correction was used in all cases. Spin-polarization did not affect the trends presented here, and was not included. The plane wave cutoff was $350 \mathrm{eV}$ for $\mathrm{H}$, and $450 \mathrm{eV}$ for $\mathrm{O}$ adsorption calculations.

\section{Experimental}

In Table I we include a collection of experimental data for exchange currents for hydrogen evolution. We have included both the polycrystalline data as well as data from single crystal experiments. There are some variations from one measurement to the next on the same metal, but clearly the trends are the same no matter which measurement is used.

\section{Volcano Curve}

We are now in a position to generate a plot of the experimental exchange currents as a function of the calculated hydrogen chemisorption energies. We use the low coverage chemisorption energies for all metals. We will return to the effect of coverage later. The result is shown in Fig. 1. Clearly, the data order quite nicely into a volcano. Note that all the reactive metals are on the left "leg" of the volcano and the unreactive ones, $\mathrm{Cu}, \mathrm{Ag}$, and $\mathrm{Au}$, are on the right.

\section{Kinetics and the Origin of the Volcano}

The total hydrogen evolution reaction can be written

$$
\mathrm{H}^{+}+\mathrm{e}^{-} \rightarrow 1 / 2 \mathrm{H}_{2}
$$

It takes place at an electrode supplying the electrons, and providing an intermediate state of the process:

$$
\begin{gathered}
\mathrm{H}^{+}+\mathrm{e}^{-}+* \rightarrow \mathrm{H}^{*} \\
2 \mathrm{H}^{*} \rightarrow \mathrm{H}_{2}
\end{gathered}
$$

where the $*$ denotes a site on the surface (so an $*$ by itself denotes a free site and $\mathrm{H}^{*}$ denotes a hydrogen atom adsorbed on the surface). The final hydrogen evolution step may also be

$$
\mathrm{H}^{+}+\mathrm{e}^{-}+\mathrm{H}^{*} \rightarrow \mathrm{H}_{2}
$$

We now consider hydrogen evolution at a metal electrode, which is part of an electrochemical cell. During hydrogen evolution a current $i$ will be running

$$
i=-e r
$$

where $r=r_{+}-r_{-}$is the net rate of (Eq. 2).

The exchange current is the forward (and backward) rate when Eq. 2 is in equilibrium 


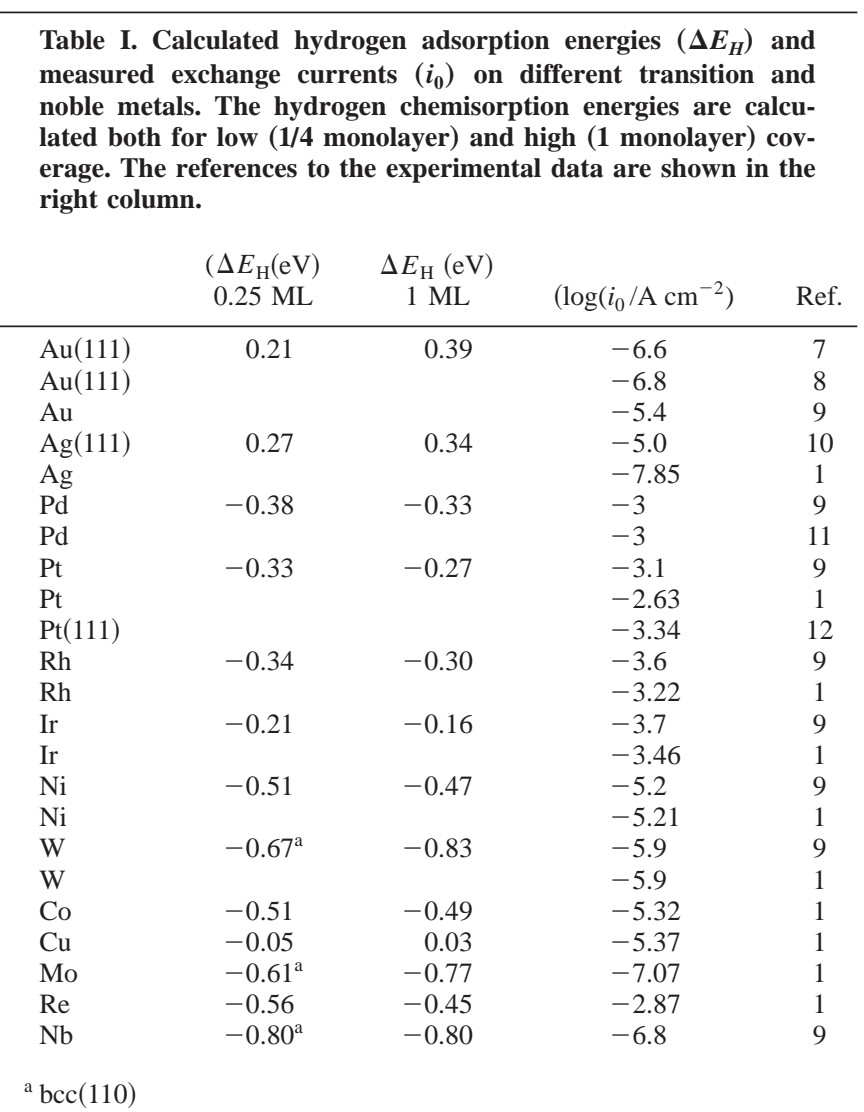

$$
i_{0}=\left.i_{+}\right|_{\mathrm{eq}}=\left|i_{-}\right|_{\mathrm{eq}}=-e r_{0}
$$

The free energy diagrams for the reaction at equilibrium over $\mathrm{Pt}(111), \mathrm{Ni}(111), \mathrm{Mo}(110)$, and $\mathrm{Au}(111)$ are shown in Fig. 2. At equilibrium the free energy per $\mathrm{H}$ atom (the chemical potential) of the initial and final states of reaction (Eq. 2) are the same. In Fig. 1 and 2 we have also included the free energy of the adsorbed state calculated as

$$
\Delta G_{H^{*}}=\Delta E_{\mathrm{H}}+\Delta E_{\mathrm{ZPE}}-T \Delta S_{\mathrm{H}}
$$

where $\Delta E_{\mathrm{H}}$ is the hydrogen chemisorption energy from Table I and $\Delta E_{\mathrm{ZPE}}$ is the difference in zero point energy between the adsorbed and the gas phase. We exploit the fact that the vibrational entropy in the adsorbed state is small meaning that the entropy of adsorption of $1 / 2 \mathrm{H}_{2}$ is $\Delta S_{\mathrm{H}} \cong-1 / 2 S_{\mathrm{H}_{2}}^{0}$, where $S_{\mathrm{H}_{2}}^{0}$ is the entropy of $\mathrm{H}_{2}$ in the gas phase at standard conditions. $\Delta E_{\mathrm{ZPE}}$ is calculated to be $0.04 \mathrm{eV}$ for $\mathrm{H} / \mathrm{Cu}(111)$, see the Appendix, ${ }^{13}$ and we take this value to be representative for all the metals we study here. This means that $\Delta G_{\mathrm{H}^{*}}=\Delta E_{\mathrm{H}}+0.24 \mathrm{eV}$.

In Fig. 2 we have chosen to show data for low coverage (1/4 monolayer). As indicated in Table I, trends will not change if we include the possibility of a higher coverage. The figure indicates that over $\mathrm{Pt}$ the reaction is essentially thermo-neutral where as this is not the case for metals on either side of the volcano, e.g., $\mathrm{Ni}, \mathrm{Au}$, and Mo. This in itself indicates why $\mathrm{Pt}$ is a much better electrocatalyst than the other metals for hydrogen evolution at the equilibrium potential.

We now turn to the kinetic modelling. We will concentrate on the trends in exchange rates from one metal to the next and on the contribution to these trends originating directly from the variations in the thermochemistry, Fig. 2 and Table II. We therefore neglect contributions to the variation in the rate constants from any reaction barriers other than those coming from the thermochemistry. This means that the variations from one metal to the next in the rate

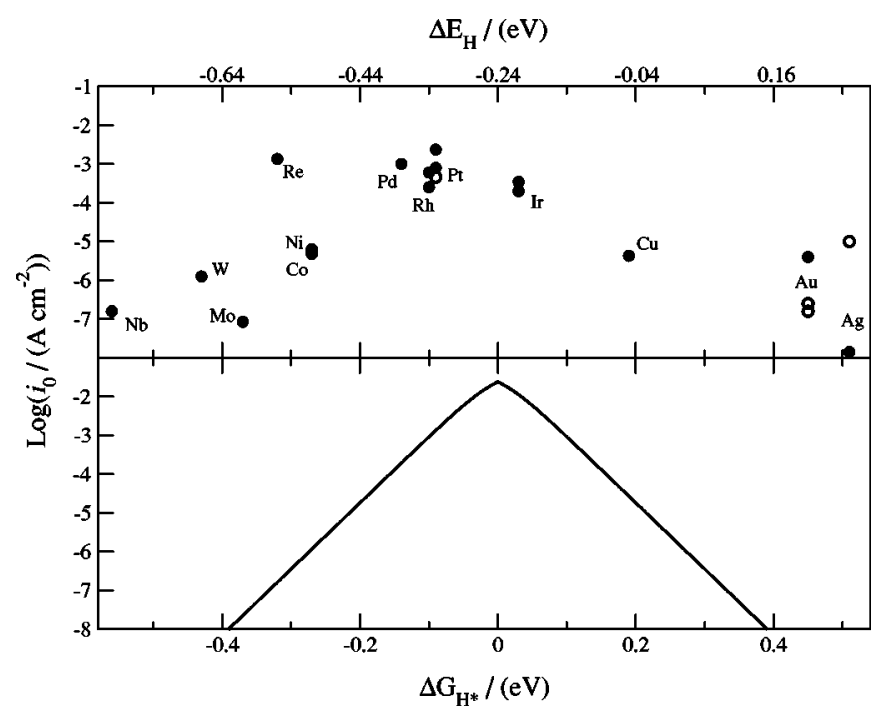

Figure 1. (top) Experimentally measured exchange current, $\log \left(\mathrm{i}_{0}\right)$, for hydrogen evolution over different metal surfaces plotted as a function of the calculated hydrogen chemisorption energy per atom, $\Delta \mathrm{E}_{\mathrm{H}}$ (top axis). All data are shown in Table I. Single crystal data are indicated by open symbols. (bottom) The result of the simple kinetic model now plotted as a function of the free energy for hydrogen adsorption, $\Delta G_{\mathrm{H}^{*}}=\Delta E_{\mathrm{H}}+0.24 \mathrm{eV}$, Eq. 8 .

constants for getting out of the chemisorption state, $\mathrm{H}^{*}$ in Fig. 2, are the same. Combined with the fact that at equilibrium the rates involved in the forward and backward steps must be the same, it means that the exchange rates of the two consecutive steps are the same. We choose here to focus on the rate of the proton reduction, Eq. 3. The forward rate of Eq. 3 can be written

$$
r_{1}=k_{1}(1-\theta) c_{\mathrm{H}^{+}}
$$

where $k_{1}$ is the rate constant, $\theta$ is the $\mathrm{H}$ coverage (measured in fraction of a monolayer with respect to the number of metal atoms in the surface layer), and $c_{\mathrm{H}^{+}}$is the concentration of protons in the electrolyte. The coverage of hydrogen can be calculated from Eq. 4, and hence

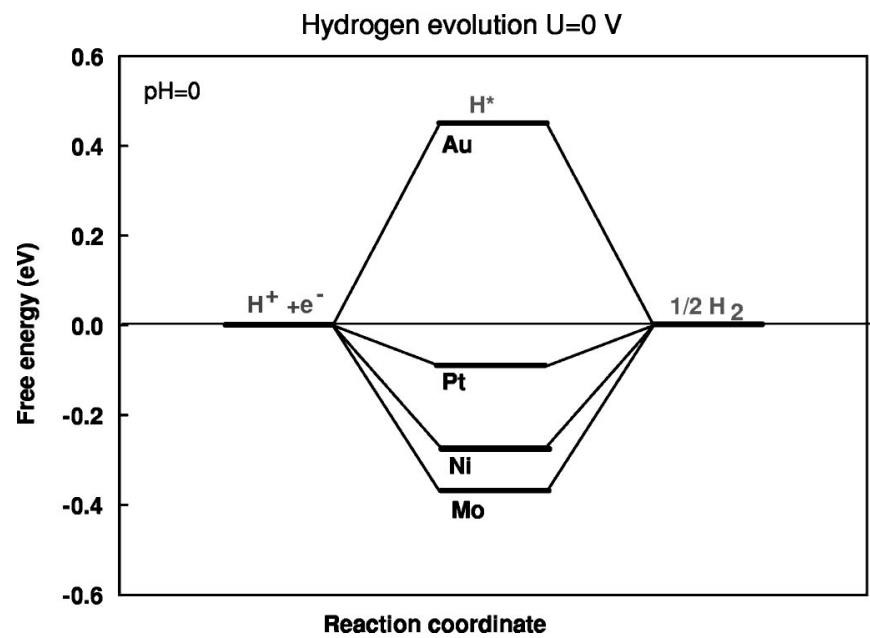

Figure 2. Free energy diagram for hydrogen evolution at equilibrium $(\mathrm{U}=0)$. The data are for "standard" conditions corresponding to 1 bar of $\mathrm{H}_{2}$ and $\mathrm{pH}=0$ at $300 \mathrm{~K}$. The energies for the intermediate adsorbed state are from the DFT calculations shown in Table I, corrected for entropy and zero point energies as indicated in the text. Data for adsorbate coverages of $1 / 4$ are used here. 


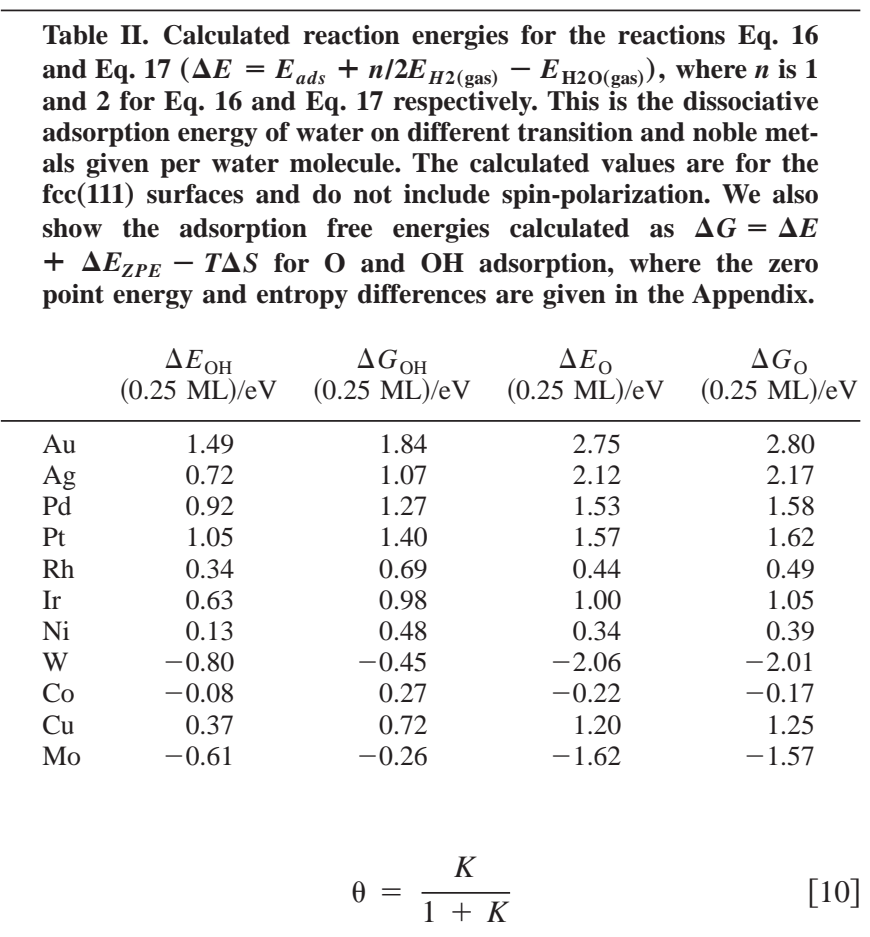

where

$K=\exp \left(-\Delta G_{\mathrm{H}^{*}} / k T\right)=\exp \left(-\left(\Delta E_{\mathrm{H}}+\Delta E_{\mathrm{ZPE}}+1 / 2 T S_{\mathrm{H}_{2}}^{0}\right) / k T\right)$

Let us first consider the limit where the proton transfer is exothermic $\left(\Delta G_{\mathrm{H}^{*}}<0\right)$. In this case we would expect the rate constant $k_{1}=k_{0}$ to be large and independent of $\Delta G_{\mathrm{H}^{*}}$. The rate constant $k_{0}$ includes all effect relating to the reorganization of the solvent during the proton transfer to the surface, and we are thus assuming this to be independent of the metal at the equilibrium potential. This leads to the following expression for the exchange current at $\mathrm{pH} 0$

$$
i_{0}=-e k_{0} \frac{1}{1+\exp \left(-\Delta G_{\mathrm{H}^{*}} / k T\right)}
$$

For the other case where the proton transfer is endothermic ( $\Delta G_{\mathrm{H}^{*}}>0$, e.g., Au in Fig. 2), we would expect the proton transfer to be activated by at least $\Delta G_{\mathrm{H}^{*}}$. Under the same assumptions as above, the rate constant for this case is

$$
k_{1}=k_{0} \exp \left(-\Delta G_{\mathrm{H}^{*}} / k T\right)
$$

and the exchange current is

$$
i_{0}=-e k_{0} \frac{1}{1+\exp \left(-\Delta G_{\mathrm{H}^{*}} / k T\right)} \exp \left(-\Delta G_{\mathrm{H}^{*}} / k T\right) .
$$

The result of the model, with the single unknown parameter $k_{0}=200 \mathrm{~s}^{-1}$ site $^{-1}$ fitted to give a reasonable overall magnitude of the rate is included in Fig. 1. The trends are well described by the simple model, including in particular the position of the maximum. Clearly $\Delta G_{\mathrm{H}^{*}}=0$ separates the two legs of the volcano. Such a picture has been suggested before, ${ }^{2}$ and our calculated database of chemisorption energies puts the physical picture on a firm footing.

Note that the model seems to underestimate the current density for the metals furthest away from the maximum. This can be understood in the following way. For the most noble metals Ag and Au, steps and other low coordination number defects will have a stronger metal-hydrogen bond than the close packed surfaces. ${ }^{14}$ These defects will therefore be closer to the maximum and may give an

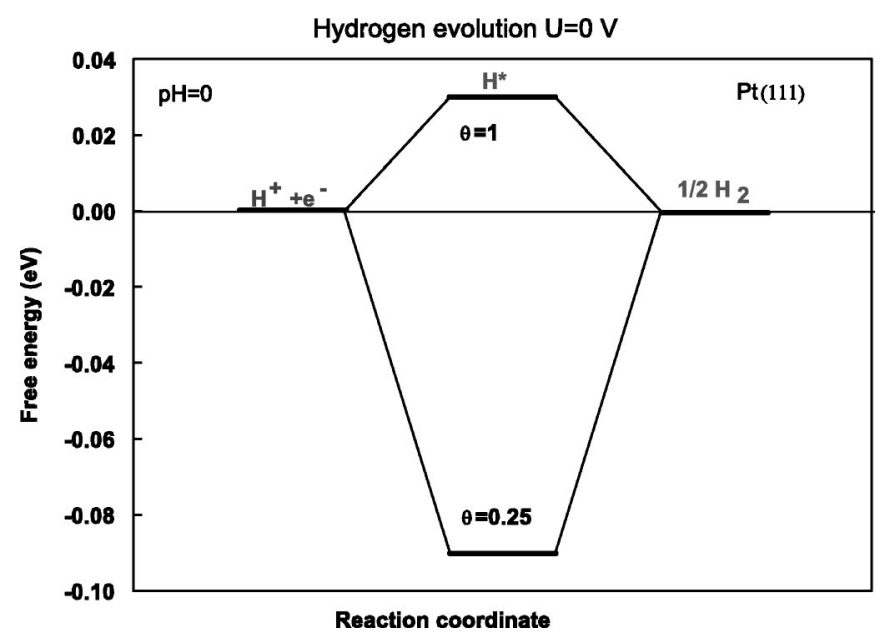

Figure 3. Free energy diagram for hydrogen evolution over $\operatorname{Pt}(111)$ at two different hydrogen coverages, $\theta=0.25$ and $\theta=1$ monolayers. At $\theta=1$ we use the differential heat of adsorption as defined in Eq. 15.

extra contribution to the current. For the least noble metals, Mo, W, and $\mathrm{Nb}$, the surface is covered by an oxide (see later) and the measured values are most probably not representative of the metal in the metallic state.

The results indicate that we can understand the two branches of the volcano curve in the following way: To the left of the maximum, the rate decreases with decreasing $\Delta E_{\mathrm{H}}$ due to a lack of available sites for $\mathrm{H}+\mathrm{H}$ recombination at the surface. Here hydrogen bonds too strongly. To the right of the maximum, the rate decreases with increasing $\Delta E_{\mathrm{H}}$ because proton transfer becomes more and more difficult as hydrogen becomes more and more unstable on the surface. Here hydrogen bonds too weakly. Pt is very close to optimum, because all reaction steps of the hydrogen evolution process on this metal are thermo-neutral.

There has been other suggested volcano curves for the hydrogen evolution reaction. ${ }^{1,2}$ Our volcano distinguishes itself from these in using a systematic database for the intrinsic H-metal interaction energy as the descriptor (or x-axis). This provides an extremely simple picture of the origin of the volcano, as outlined above.

In Fig. 1 and 2 we have used the low coverage adsorption energies from Table I. Clearly, the high coverage energies follow exactly the same trends and thus give the same picture. However, one point about the coverage dependence deserves further comments. In Fig. 3 we show the free energy diagram for two different coverages of hydrogen on $\mathrm{Pt}(111)$. We use a low coverage value, which we take from the $\theta=0.25$ result in Table I. At $\theta=1$ we use the differential heat of adsorption. For simplicity we consider here a two-state model with only a low coverage and a high coverage state, and the differential heat of adsorption in the high coverage state is the energy difference between two hydrogens in the high coverage state and one in the low coverage state

$$
\Delta E_{\mathrm{H}}^{\mathrm{diff}}(1)=2 \Delta E_{\mathrm{H}}(1)-\Delta E_{H}(0.25)
$$

Figure 3 suggests the following physical picture. At equilibrium, the low coverage state is fully occupied. The addition of a new proton and electron must add a hydrogen atom in the high coverage state. Both states can turn over, but the overall activation energy is smaller for the high coverage state, making this the most likely candidate for the catalytically active species. This could be an explanation of the two types of hydrogen usually invoked to explain experimental data in the electrochemistry literature. ${ }^{2}$

\section{Water Dissociation on the Surface}

One question in connection with the experimental data in Table I and Fig. 1 is whether reaction products from the adsorption and 
dissociation of water might cover some of the metals. To investigate this we have first calculated the effect of molecular water on the adsorption. The result is that adding a water overlayer changes the adsorption energy of hydrogen on Pt(111) by less than $0.02 \mathrm{eV}$. Even though the effect could be a little larger for the more reactive metals it seems justified to neglect it in the present trend study.

In addition we have calculated the reaction energies for the reactions

$$
\mathrm{H}_{2} \mathrm{O}+* \rightarrow \mathrm{OH}^{*}+1 / 2 \mathrm{H}_{2}
$$

and

$$
\mathrm{H}_{2} \mathrm{O}+* \rightarrow \mathrm{O}^{*}+\mathrm{H}_{2}
$$

The results are included in Table II. In general one should also consider the possibility of forming solvated $\mathrm{H}^{+}$and electrons at the electrode rather than $\mathrm{H}_{2}$, but for the exchange current at standard conditions this makes no difference since the solvated hydrogen is in equilibrium with $\mathrm{H}_{2}$ (gas). The values in Table II are relative to gas phase water. We also include the adsorption free energies, calculated using the zero point energies and entropies collected in the Appendix. The result is that of the metals considered in Table II, only Mo and $\mathrm{W}$ should be covered by surface oxygen under the conditions of interest here. This means that the measured exchange currents for these metals in Fig. 1 should not be taken to be representative of the metal, but rather of the surface oxide.

\section{Conclusions}

The results presented above clearly demonstrate the correlation between the hydrogen chemisorption energies and the exchange current for hydrogen evolution. The volcano curves in Fig. 1 also indicate that $\mathrm{Pt}$ is a better electrocatalyst than other metals for hydrogen evolution, primarily because the evolution reaction is thermo-neutral on $\mathrm{Pt}$ at the equilibrium potential. These findings can potentially be useful in predicting bimetallic electrocatalysts for hydrogen evolution, as well as for hydrogen electro-oxidation for fuel cell applications.

\section{Acknowledgments}

Useful discussions with J. Meier and A. K. Pal are appreciated. This work was funded in part by U.S. Army Research Office (ARO), USA, under grant DAAD19-02-1-0311 (U.S. and S.P.). The DFT calculations have been performed with support from the Danish Center for Scientific Computing through grant no. HDW-1101-05. A.L. acknowledges financial support from the European Union through contract no. ENK5-CT-2001-00572.

The Technical University of Denmark assisted in meeting the publication

\begin{tabular}{|c|c|c|c|c|}
\hline & $\begin{array}{c}T S \\
(\mathrm{eV})\end{array}$ & $\begin{array}{l}T \Delta S \\
(\mathrm{eV})\end{array}$ & $\begin{array}{l}E_{\mathrm{ZPE}} \\
(\mathrm{eV})\end{array}$ & $\begin{array}{c}\Delta E_{\mathrm{ZPE}} \\
(\mathrm{eV})\end{array}$ \\
\hline $\mathrm{H}_{2} \mathrm{O}(0.035$ bar $)$ & 0.67 & 0 & 0.56 & 0 \\
\hline$* \mathrm{OH}+1 / 2 \mathrm{H}_{2}$ & 0.20 & -0.47 & 0.44 & -0.12 \\
\hline$* \mathrm{O}+\mathrm{H}_{2}$ & 0.41 & -0.27 & 0.34 & -0.22 \\
\hline $1 / 2 \mathrm{O}_{2}+\mathrm{H}_{2}$ & 0.73 & 0.05 & 0.32 & -0.24 \\
\hline $\mathrm{H}_{2}$ & 0.41 & $\cdots$ & 0.27 & $\cdots$ \\
\hline $1 / 2 \mathrm{O}_{2}$ & 0.32 & $\cdots$ & 0.05 & $\cdots$ \\
\hline $\mathrm{O}^{*}$ & 0 & $\ldots$ & 0.07 & $\cdots$ \\
\hline $\mathrm{OH}^{*}$ & 0 & $\cdots$ & 0.30 & $\cdots$ \\
\hline $\mathrm{H}^{*}$ & 0 & & 0.17 & \\
\hline
\end{tabular}
costs of this article.

\section{Appendix}

In Table A-1 we show the entropies and zero point energies (ZPEs) used in the construction of the free energies in Fig. 2 and 3 and in Table II. The gas phase values are from Ref. 15, while the values for the adsorbed species are taken from DFT calculations for $\mathrm{O}$ and $\mathrm{OH}$ adsorbed on $\mathrm{Cu}(111)$ by Gokhale et al. ${ }^{13}$ We use gas phase $\mathrm{H}_{2} \mathrm{O}$ at 0.035 bar as the reference state because at this pressure gas phase $\mathrm{H}_{2} \mathrm{O}$ is in equilibrium with liquid water at $300 \mathrm{~K} .{ }^{16}$ We use the same values for the adsorbed species for all the metals, as vibrational frequencies have been found to depend much less on the metal than the bond strength. ${ }^{17}$

\section{References}

1. S. Trasatti, J. Electroanal. Chem., 39, 163 (1972).

2. J. O'M Bockris, A. K. N. Reddy, and M. Gamboa-Aldeco, Modern Electrochem istry, 2nd ed., p. 1285, Kluver Academic/Plenum Publishers, New York (2000).

3. M. C. Payne, M. P. Teter, D. C. Allan, T. A. Arias, and J. D. Joannopoulos, Rev. Mod. Phys., 64, 1045 (1992).

4. G. Kresse and J. Furtmüller, Comput. Mater. Sci., 6, 15 (1996).

5. D. Vanderbilt, Phys. Rev. B, 41, 7892 (1990).

6. B. Hammer, L. B. Hansen, and J. K. Nørskov, Phys. Rev. B, 46, 7413 (1999).

7. J. Perez, E. R. Gonzalez, and H. M. Villullas, J. Phys. Chem. B, 102, 10931 (1998).

8. S. Pandelov, A. K. Pal, J. Meier, and U. Stimming, Unpublished results.

9. J. O'M. Bockris and A. K. N. Reddy, Modern Electrochemistry, Vol. 2, Plenum Press, New York (1970).

10. D. Eberhardt, E. Santos, and W. Schmickler, J. Electroanal. Chem., 461, 76 (1999).

11. O. Savadogo and D. L. Piron, Int. J. Hydrogen Energy, 15, 715 (1990).

12. N. M. Markovic and P. N. Ross, in Interfacial Electrochemistry, A. Wieckowski, Editor, Marcel Dekker, New York (1999).

13. A. A. Gokhale, J. A. Dumesic, and M. Mavrikakis, Unpublished results.

14. T. Bligaard, J. K. Nørskov, S. Dahl, J. Matthiesen, C. H. Christensen, and J. Sehested, J. Catal., 224, 206 (2004).

15. P. W. Atkins, Physical Chemistry, 6th ed., p. 485, 925, and 942, Oxford University Press, Oxford (1998).

16. CRC Handbook of Chemistry and Physics, 49th ed., Robert C. Weast, Editor, p. D109, The Chemical Rubber Company, Cleveland (1969).

17. T. Bligaard, K. Honkala, A. Logadottir, J. K. Nørskov, S. Dahl, and C. J. H. Jacobsen, J. Phys. Chem. B, 107, 9325 (2003). 